\title{
DESENVOLVIMENTO DOS FRUTOS DE PÊSSEGO 'AURORA' E NECTARINA 'SUNRAYCER' NO SUL DE SANTA CATARINA'
}

\author{
EMILIO DELLA BRUNA² \& ALEXSANDER LUIS MORETO
}

RESUMO - O desenvolvimento do fruto de pessegueiro é resultado da diferenciação e do crescimento das paredes do ovário após a fecundação. A persistência e o crescimento do fruto na planta dependem das relações exatas entre os hormônios auxina, giberelina e citocinina que condicionam o desenvolvimento dos frutos, caracterizado por uma curva dupla sigmoide, com três estádios distintos. O presente trabalho teve por objetivo conhecer o comportamento dos frutos e das sementes do pêssego Aurora e da Nectarina Sunraycer durante todo o seu ciclo de desenvolvimento. $\mathrm{O}$ crescimento dos frutos e das sementes durante o ciclo foi determinado semanalmente, coletando-se 30 frutos de dez diferentes plantas em ramos previamente identificados. As sementes foram separadas do fruto para a determinação do peso fresco (PF) e do peso seco (PS). O crescimento dos frutos da variedade Sunraycer dá-se de forma contínua e acelerada desde a floração até a maturação, sugerindo um curto período ou a inexistência do Estágio II de crescimento. Para a variedade Aurora, a curva de crescimento é diferenciada nos três estádios (I, II e III). O raleio dos frutos deve ser feito até o início do estádio II, para a variedade Aurora e Sunraycer. As sementes das variedades Aurora e Sunraycer atingem seu tamanho máximo no estágio I de crescimento do fruto. O aumento de peso seco na semente, para a variedade Sunraycer, é praticamente inexistente no estádio III, enquanto para a variedade Aurora ocorre o maior aumento de peso seco que vai até a maturação do fruto.

Termos para indexação: Curva de crescimento, Produção, Ecofisiologia.

\section{DEVELOPMENT OF THE 'AURORA' PEACH AND 'SUNRAYCER' NECTARINE FRUITS IN SOUTHERN SANTA CATARINA, BRAZIL}

\begin{abstract}
The peach fruit development is a result of the differentiation and growth of the ovary wall after fertilization. The persistency and the growth of the fruit on the plant depend on the exact relationships among auxin, gibberelin and cytokinin hormones, which condition the development of the fruits, characterized by a double sigmoid curve with three distinct stages. This study aimed to understand the performance of fruits and seeds of the Aurora peach and Sunraycer nectarine throughout their development cycle. Fruits and seeds growth was evaluated weekly by collecting 30 fruits from previously identified branches of ten different plants. The seeds were separated from the fruit for the determination of the fresh weight (FW) and the dry weight (DW). The growth of the Sunraycer fruits variety is continuous and accelerated from flowering to maturity, which suggests a short period or absence of the growth Stage II. For the Aurora variety, the growth curve is differentiated during the three stages (I, II and III). The fruits thinning must be done until the beginning of stage II for both varieties, Aurora and Sunraycer. Their seeds reach their maximum size at stage I of fruit growth. The increase in the dry weight of the Sunraycer variety was practically absent on stage III, whereas it increased significantly in the Aurora variety until the fruit maturation.
\end{abstract}

Index terms: Growth curve, Production, Ecophisiology.

\section{INTRODUÇÃO}

A área plantada com pêssego no Brasil, em 2009, era de 20.000 ha, distribuída nos Estados do Rio Grande do Sul (14.617 ha), Paraná (1.555 ha), São Paulo (1.416 ha), Minas Gerais (960 ha), Santa Catarina (457 ha), Espírito Santo (21 ha) e Rio de Janeiro (17 ha) (IBGE, 2011). Embora grande parte da área plantada esteja localizada no Estado do Rio Grande do Sul, os demais Estados possuem um grande potencial para a expansão do plantio. A proximidade com o mercado consumidor e a criação de novas variedades de baixa exigência em frio e com frutos de qualidade, desenvolvidas pelos programas de melhoramento genético da Embrapa-CPACT e da Epagri-EE Urussanga, são fatores que devem

\footnotetext{
${ }^{1}$ Trabalho Sinfruit 051 - Simpósio Internacional de Fruticultura - Avanços na Fruticultura (17 a 21 Outubro)

${ }^{2}$ Eng $^{\circ}$ Agrônomo MS. Epagri - Estação Experimental de Urussanga, C.P. 48, Cep 88840-000. E-mail: emilio@epagri.sc.gov.br

${ }^{3}$ Eng $^{\mathrm{o}}$ Agrônomo Dr. Epagri - Estação Experimental de Urussanga, C.P. 48, Cep 88840-000. E-mail: alexsandermoreto@epagri.sc.gov.br
} 
impulsionar a persicultura nos demais estados do Centro-Sul do País. As variedades de pessegueiro e nectarineira recomendadas para cultivo em regiões subtropicais apresentam ciclo entre a floração e a maturação dos frutos, de 74 e até 181 dias, podendo ser classificados em ultraprecoces, precoces, medianos, tardios e bem tardios (BARBOSA et al., 1990).

Nas regiões de clima subtropical, o cultivo do pessegueiro requer técnicas diferenciadas de manejo da planta, tais como a aplicação de cianamida hidrogenada para a quebra de dormência e a realização de podas auxiliares, especialmente poda verde. (PEREIRA; MAYER, 2008). Nessas regiões, as plantas vegetam por um longo período após a colheita dos frutos, exigindo manejo fitossanitário e adubações adequadas para que a planta acumule reservas para a próxima safra e, ao mesmo tempo, mantenha a vegetação equilibrada.

$\mathrm{O}$ desenvolvimento do fruto de pessegueiro é o resultado da diferenciação e do crescimento das paredes do ovário após a fecundação (BARBOSA et al., 1993). A persistência e o crescimento do fruto na planta dependem das relações exatas entre auxinas, giberelinas e citocininas (POWELL; PRATTI, 1966, VALPUESTA et al., 1989). Esses hormônios condicionam o desenvolvimento dos frutos caracterizado por uma curva sigmoidal dupla, com três estádios distintos (BARBOSA et al., 1993; CUNHA JÚNIOR et al., 2007; DELA BRUNA, 2007). Durante o estádio I do desenvolvimento, há um rápido crescimento, devido à divisão celular. Já no estádio II, verificam-se importantes mudanças fisiológicas e anatômicas do fruto, como: diminuição do ritmo de crescimento da polpa, com endurecimento do caroço, e formação parcial ou total da semente. No estádio III, a polpa retoma seu crescimento, aumentando os volumes celulares e os espaços intercelulares (CRANE, 1969; VÁLIO, 1979; FELIPPE, 1979; KING et al., 1987). Inúmeras pesquisas foram desenvolvidas para explicar os mecanismos que controlam as diferentes taxas de crescimento dos frutos, geralmente correlacionadas com o desenvolvimento do caroço (pericarpo), da semente e com a atividade vegetativa da planta (TUKEY, 1936; CHALMERS; VAN DEN EDEN ,1975 , 1977; BARBOSA ET AL.,1993; GOMES et al., 2005). Os fatores endógenos e ambientais que controlam o crescimento e o desenvolvimento dos frutos também foram estudados por Batjer e Martin (1965), Quilot et al. (2005) e Haun e Coston (1983). Já Fischer (1962) e DeJong e Goudriaan (1989) mostraram que o acúmulo de unidade de calor ('Grausdia') tem sido uma medida eficiente para medir o tempo de desenvolvimento das frutas de caroço. A maioria dos estudos sobre o desenvolvimento dos frutos tem sido feita considerando-se o diâmetro e o peso fresco dos frutos. Entretanto, Chalmers e Van Den Eden (1977), analisando o crescimento do fruto nos três estágios de desenvolvimento, observaram que o peso seco do fruto nem sempre corresponde ao seu peso fresco.

O presente trabalho teve por objetivo conhecer o desenvolvimento dos frutos e das sementes do pêssego Aurora e da Nectarina Sunraycer durante todo o seu ciclo, facilitando assim a recomendação das práticas culturais que levem à produção de frutos de alta qualidade.

\section{MATERIAL E MÉTODO}

O experimento foi conduzido em pomares comerciais de produtores associados à Associação dos Fruticultores do Sul de Santa Catarina (SULFRUTAS), localizados no Município de Urussanga - SC (283' $40^{\prime \prime}$ 'S e 49 $9^{\circ} 19^{\prime} 30^{\prime \prime} \mathrm{O}$ ), com plantas de seis anos de idade, enxertadas sobre o porta-enxerto Okinawa, cultivadas em solo Argissolo, com clima subtropical úmido, com verão quente (cfa), pela classificação de Köeppen, com temperatura média anual de $19,4^{\circ} \mathrm{C}$, variando de $14,6^{\circ} \mathrm{C}$ em julho a $24,1^{\circ} \mathrm{C}$ em fevereiro (PANDOLFO et al., 2002). Foram selecionadas dez plantas de cada variedade e, para cada planta, foram identificados quinze ramos de produção com aproximadamente 70 centímetros de comprimento. Cada ramo foi identificado para uma data de coleta, totalizando quinze datas de coleta repetidas em cada uma das dez plantas. As amostragens dos frutos para análise iniciaram dez dias após a plena floração (DAF) e repetiram-se a cada sete dias até a maturação do fruto. Para as duas primeiras amostragens, aos 10 e aos 17 dias DAF, foram determinados somente o peso fresco (PF) e o peso seco (PS) dos frutos. A partir do $24^{\circ}$ dia DAF, determinaram-se o PF e o PS do fruto e da semente, separadamente. Para a determinação do PF e do PS, os frutos foram coletados, transportados em sacos plásticos e pesados inteiros; após, separaram-se as sementes dos frutos que foram pesadas, determinando-se assim o PF dos frutos e das sementes. Os frutos e as sementes, separadamente, foram secados em estufa até peso constante para a determinação do PS (MAGALHÃES, 1979).

\section{RESULTADOS E DISCUSSÃO}

O período entre a floração e a maturação foi de 87 e 108 dias para a nectarina Sunraycer e para o pêssego Aurora, respectivamente, podendo ser classificados como de ciclo precoce e médio (DELA BRUNA, 2007). O pessegueiro, dependendo de suas 
características genéticas, podem apresentar ciclos de desenvolvimento bastante diferenciados (TUKEY, 1933; LILLIEN-KIPNIS; LAVEE, 1971). Barbosa et al. (1993) descrevem que, no Estado de São Paulo, com clima subtropical, há variedades desde precoces até tardias, com ciclos de 80 a 200 dias. Além dos fatores genéticos, a temperatura expressa em Graus-dia também influencia no ciclo dos frutos (GROSSMAN; DEJONG, 2005). Para a variedade Aurora, o ciclo pode variar de 82 dias na região de Jaboticabal (PEREIRA; MAYER, 2008) até 115 na região de Jundiaí (BARBOSA et al., 1990).

Os dados das Figuras 1A e 1B mostram a curva de crescimento dos frutos da variedade Aurora caracterizada por uma curva dupla sigmoide, diferenciando claramente os estádios de desenvolvimento I, II e III. Para a variedade Sunraycer, de ciclo precoce, a curva de crescimento não mostra o estádio II, que é caracterizado pela redução do crescimento e do acúmulo de matéria seca. Resultados semelhantes foram obtidos por DeJong e Goudriaan (1989), que, estudando a influência da temperatura no desenvolvimento dos frutos de pessegueiro, também observaram que a variedade June Lady, com ciclo de aproximadamente 100 dias, apresentou um curto Estágio II. Dela Bruna (2007), estudando o crescimento de frutos de pessegueiro cultivados em regiões subtropicais, observou que a curva de crescimento para as variedades precoces não apresenta o estádio II de crescimento, apresentando um crescimento acelerado, desde a floração até a maturação. Dela Bruna (2007) também observou que o período de duração do estádio II é maior à medida que aumenta o ciclo do fruto. Na Figura 1C observamos o percentual de matéria seca (MS) existente nos frutos das duas variedades estudadas. A variedade Aurora, durante todo o seu ciclo, apresentou maior teor de matéria seca (MS) que a variedade Sunraycer. Para as duas variedades, o maior teor de MS ocorreu aos $38 \mathrm{DAF}$. No estádio de maturação meio-maduro (MEDEIROS; RASEIRA, 1998), que corresponde a $101 \mathrm{DAF}$, a variedade Aurora apresentou um aumento no teor de MS que caiu novamente no ponto de colheita. A duração do estádio I foi de aproximadamente 52 dias para as duas variedades (Figura 1). A partir dos 52 até 87 DAF, a variedade Sunraycer iniciou um rápido aumento do peso fresco do fruto e diminuição do teor da MS até a maturação (Figuras 1C e 2A), caracterizando assim o estádio III de crescimento, em que ocorre a redução da firmeza com o amaciamento da polpa (CUNHA
JÚNIOR et al., 2007), formação dos aromas e transformação dos sólidos em açúcares simples, como a glicose, frutose e sacarose, gerando o aumento do teor de sólidos solúveis e açúcares solúveis (CHITARRA; CHITARRA, 2005). A variedade Aurora apresentou o estádio II de crescimento que durou 14 dias, dos 52 aos 66 DAF, quando o crescimento diário de peso fresco e peso seco foi muito baixo (Figuras 2A e 2B). Nas Figuras 1A e 1B, podemos observar, para o pêssego Aurora, o rápido acúmulo de peso fresco e peso seco no fruto, no período de 94 a108 dias. $\mathrm{O}$ peso fresco dos frutos nas últimas três semanas aumentaram de peso, de $46 \mathrm{~g}$ para $109 \mathrm{~g}$, e valores semelhantes também foram encontrados por Pereira et al. (2002).

A Figura 3 mostra o crescimento das sementes das variedades Aurora e Sunraycer, desde a floração até a maturação do fruto. Para ambas as variedades, o crescimento máximo da semente, expresso em peso fresco, foi alcançado aos 52 DAF (Figura 3A). Resultados semelhantes foram encontrados por Barbosa et al. (1993) que, estudando o desenvolvimento de sementes de variedades de pêssego de diferentes ciclos, cultivadas em regiões subtropicais, observaram que, para todas as variedades, o tamanho máximo da semente foi encontrado na fase de endurecimento do caroço. Os mesmos autores também observaram que as sementes de variedades de ciclo precoce obtêm pequenos ganhos de matéria seca após o endurecimento do caroço, resultado observado na variedade Sunraycer que, dos 52 DAF até a maturação do fruto, 87 DAF, obteve ganhos de peso seco insignificantes (Figura 3C). Já para a variedade Aurora, embora o peso fresco da semente não se tenha alterado ao longo do ciclo, durante o estádio III de crescimento até a maturação, $108 \mathrm{DAF}$, houve um significativo aumento no peso seco, de 0,5 para $1,1 \mathrm{~g}$, e da percentagem de MS, de 7,4 para 21,9\% (Figuras 3B e 3C). Os dados reforçam as evidências que o sistema vascular do fruto continua funcionando no estádio III, nutrindo o embrião no interior do caroço lignificado, conforme aventado por Barbosa et al. (1993). Barbosa et al. (1985), estudando a germinação de sementes de pêssego de variedades com diferentes ciclos de crescimento, mostraram que as variedades precoces apresentam baixa germinação, por isso essas variedades não devem ser usadas como planta-mãe nos programas de melhoramento genético para a germinação das sementes in vivo;nesse caso a única alternativa é usar a técnica de cultivo in vitro. 


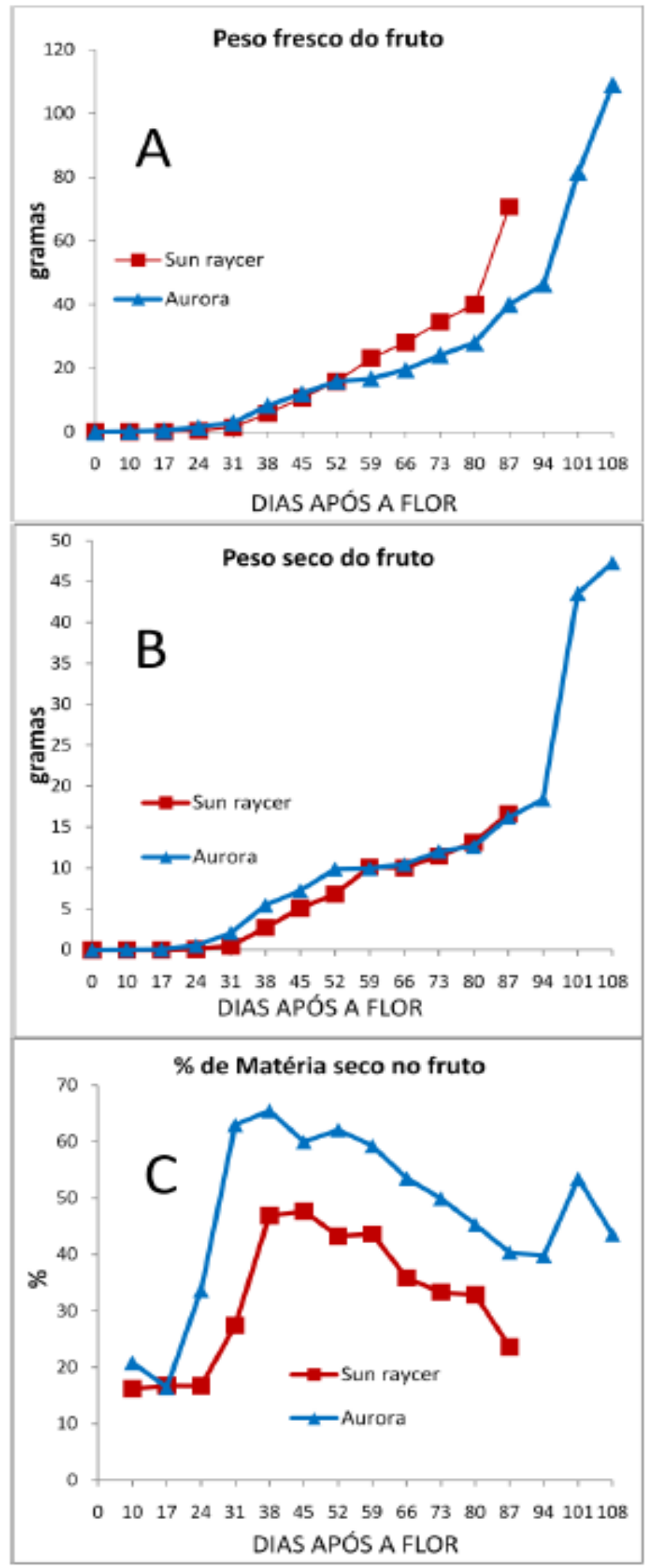

FIGURA 1 - Desenvolvimento dos frutos de pêssego Aurora e Nectarina Sunraycer. A - peso fresco expresso em gramas/fruto; B - peso seco expresso em gramas/fruto; $\mathrm{C}$ - percentagem de matéria seca no fruto. 


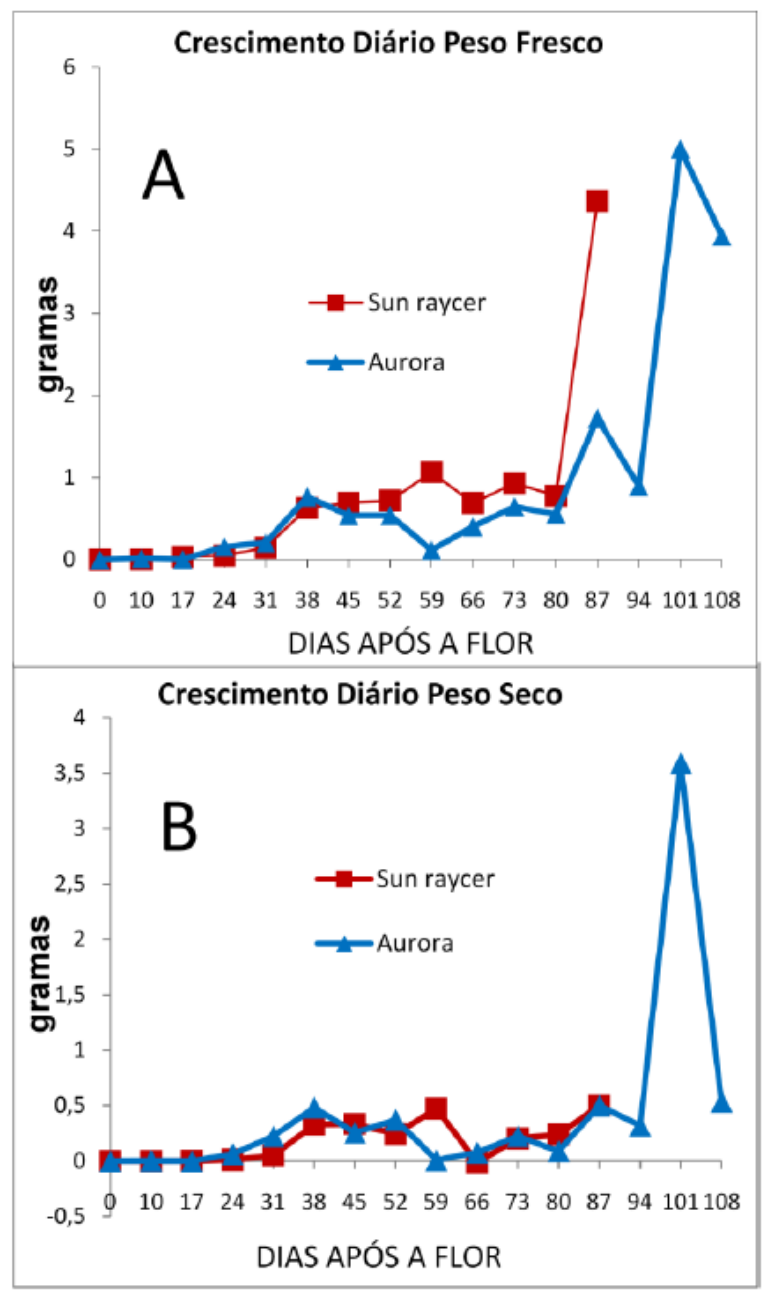

FIGURA 2 - Crescimento diário dos frutos de pêssego Aurora e de nectarina Sunraycer, expresso em gramas /dia, A - peso fresco e B - peso seco. 


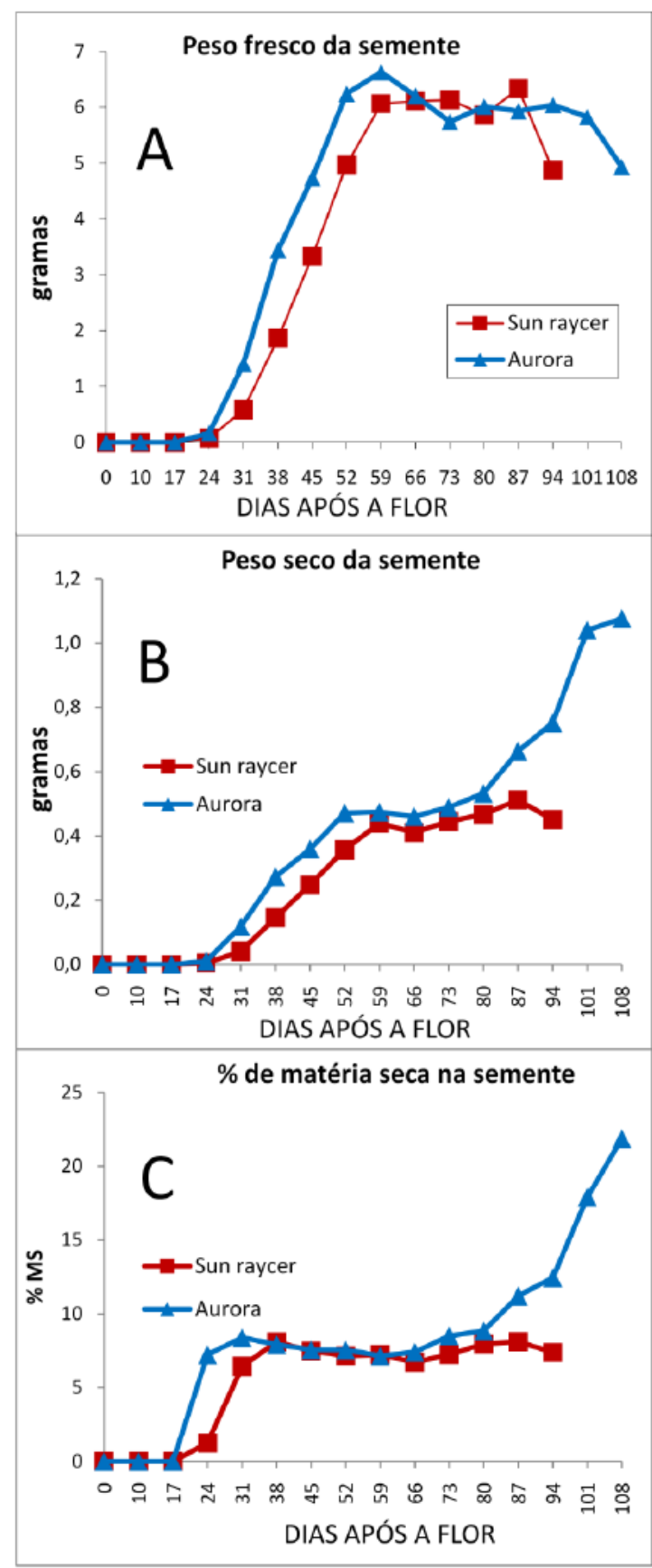

FIGURA 3 - Crescimento da semente de pêssego Aurora e de nectarina Sunraycer. A - acúumulo de peso, expresso em gramas de peso fresco/semente; $\mathrm{B}$ - acúmulo de peso seco, expresso em gramas/semente; $\mathrm{C}$ - percentagem de matéria seca na semente. 


\section{CONCLUSÃO}

1. O crescimento dos frutos da variedade Sunraycer dá-se de forma contínua e acelerada desde a floração até a maturação, sugerindo um curto período ou a inexistência do Estágio II de crescimento

2. Para a variedade Aurora, a curva de crescimento é diferenciada em três estádios (I, II e III), conforme preconizado pela literatura para variedades de ciclo médio e de ciclo longo.

3. Como a prática de raleio, objetivando a produção de frutos grandes, deve ser feita até o início do estádio II, para as variedades Aurora e Sunraycer, nas condições estudadas, deve ocorrer até 52 DAF.

4. A semente de pêssego das variedades Aurora e Sunraycer atinge seu tamanho máximo no estágio I de crescimento do fruto.

$O$ aumento de peso seco na semente, para a variedade Sunraycer, de ciclo curto, é praticamente inexistente no estádio III, enquanto para a variedade Aurora, de ciclo médio, ocorre o maior aumento de peso seco, que vai até a maturação do fruto.

\section{REFERÊNCIAS}

BARBOSA, W.; CAMPO-DALL'ORTO, F.A.; OJIMA, M. Cultura d embriões in vitro para melhoramento de pessegueiros precoces. Bragantia, Campinas, v.44, n.1, p.465-472, 1985.

BARBOSA, W.; OJIMA, M.; CAMPO DALL'ORTO, F.A.; MARTINS, F.P. Época e ciclo de maturação de pêssegos e nectarinas no Estado de São Paulo. Bragantia, Campinas, v.49, n.2, p.221-226, 1990.

BARBOSA, W.; OJIMA, M.; DALL'ORTTO, F.A.C.; MARTINS, F.P.; LOVATE, A.A. Desenvolvimento dos frutos e das sementes de pêssegos subtropicais de diferentes ciclos de maturação. Pesquisa Agropecuária Brasileira, Brasília, v. 28, n.6, p.701-707, 1993.

BATJER, L.P.; MARTIN, G.C. The influence of night temperature on growth and development of early Redhaven peaches. Proceedings of the American Society Horticultural Science, Alexandria, v.87, p.139-144, 1965.

CHALMERS, D.J. ; VAN DEN EDEN, B. The relation between seed and fruit development in peach (Prunus persica L.). Annual of Botany, New York, v.41, p.707-714, 1977.
CHALMERS, D.J. ; VAN DEN EDEN, B. A reappraisal of the growth and development of peach fruit. Australian journal of plant physiology, Melbourne, v.2, p.623-634, 1975.

CRANE, I.C. The role of hormones in fruit set and development. HortScience, Alexandria, v. 4, n. 2, p. 108-111, 1969.

CHITARRA, M.I.F.; CHITARRA, A.B. Pós-colheita de frutos e hortaliças: fisiologia e manuseio. Lavras: ESAL-FAEPE, 2005. 785p.

CUNHA JUNIOR, L. C.; DURIGAN, M. F. B.; MATTIUZ, B.; MARTINS, R. M.; DURIGAN, J. F. Caracteização da curva de maturação de pêssegos 'Aurora-1' na região de Jaboticabal-SP, Revista Brasileira de Fruticultura, Jaboticabal, v.29, n.3, p.661-665, 2007.

DEJONG, T.M.; GOUDRIAAN, J. Modeling peach fruit growth and carbohydrate requeriments: Reevaluation of the Doublesigmoid growth pattern. Journal of the American Society for Horticultural Science, Alexandria, v.114, n.5, p.800-804, 1989.

DELA BRUNA, E. Curva de crescimento de frutos de pêssego em regiões subtropicais. Revista Brasileira de Fruticultura, Jaboticabal, v.29, n.3, p.685-689, 2007.

FELIPPE, G.M. Etileno. In: FERRI, M.G. Fisiologia vegetal. São Paulo: EPU/EDUSP, 1979. v.2, p.163-192.

FISCHER, D.V. Heat units and number of days required to mature some pome end stone fruits in various areas of North America. Proceedings of the American Society for Horticultural Science, Alexandria, v.80, p.114-124, 1962.

GOMES, F.R.C.; FACHINELLO,J.C.; MEDEIROS, A.R.M.; GIACOBBO, C.L.; SANTOS, I.P. Influência do manejo do solo e intensidade de raleio de fruta, no crescimento e qualidade de pêssegos, cvs. Cerrito e Chimarrita. Revista Brasileira de Fruticultura, Jaboticabal, v.27, n.1,p.60-63, 2005.

GROSSMAN, Y.L.; DEJONG, T.M. Maximum fruit growth potential following resouce limitation during peach growth. Annals of Botany. New York. v.75, p.561-567. 2005. 
HAUN, J.R.; COSTON, D.C. Relationship of daily growth and development of peaches leaves and fruit to environmental factors. American Society Horticultural Science, Alexandria , v.108, p.666-671, 1983.

INSTITUTO BRASILEIRO DE GEOGRAFIA E ESTATÍSTICA - IBGE. Censo agropecuário 2006. Rio de Janeiro: IBGE. 2011. Disponível em: <www. ibge.gov.br/home/estatistica/economia/agropecuaria/ censoagro/2006/default.shtm $>$. Acesso em: 05 jun. 2011.

KING, G.A.; HENDERSON, K.G.; LILL, R.E. Growth and anatomical and ultrastructural studies of nectarine fruit wall development. Botanical Gazette, Chicago, v.148, n.3, p.443-455, 1987.

LILLIEN-KIPNIS, H.; LAVEE, S. Anatomical changes during the development of "Ventura" peach fruit. Journal Horticultural Science, Alexandria, v.46, p.103-110, 1971.

MAGALHÃES, A.C.N. Análise quantitativa do crescimento. In: FERRI, M.G. Fisiologia vegetal. São Paulo: EPU/EDUSP, 1979. v.1, p.333-350.

MEDEIROS, C.A.B.; RASEIRA, M.C.B. A cultura do pessegueiro. Brasília: Embrapa-SPI, 1998. 350p.

PANDOLFO, C.; BRAGA,H.J.; SILVA JÚNIOR, V.P.; MASSIGNAN, A.M.; PEREIRA, E.S.; THOMÉ, V.M.R. Atlas climatológico digital do Estado de Santa Catarina. Florianópolis: Epagri, 2002. CD-ROM.

PEREIRA, F. M. ; MAYER,N.A. Fenologia e produção de gemas em cultivares e seleções de pessegueiro na região de Jaboticabal-SP. Revista Brasileira de Fruticultura, Jaboticabal, v.30, n.1, p.043-047, 2008.
PEREIRA, F.M.; NACHTIGAL, J.C.; ROBERTO, S.R. Tecnologia para a cultura do pessegueiro em regiões tropicais e subtropicais. Jaboticabal: FUNEP, 2002. 62 p.

POWELL, L.E.; PRATTI, C. Growth promoting substances in the developing fruit of oeach (Prunus pérsica L. Batsch). Journal Horticultural Science, Ashford, v.41, p.331-346, 1966.

QUILOT, B.; KERVELLA, J.; GÉNARD, M.; LESCOURRET,F. Analysing the genetic control of peach fruit quality through an ecophysiological model combined with a QTL approach. Journal of Experimental Botany, Oxford, v.56, n.422, p.3083-3092, 2005.

TUKEY, H.B. Development of cherry and peach fruit as affected by destruction of the embryo. Botanical Gazette, Chicago, v,98, p.1-24, 1936.

TUKEY, H.B. Growth of the peach embryo in relation growth of fruit and season of ripening. Proceedings of the American Society for Horticultural Science, Alexandria, v.30, p.209-218, 1933.

VÁLIO. I.F.M. Frutificação. In: FERRI. M.G. Fisiologia vegetal. São Paulo: EPU/EDUSP, 1979. v. 2, p. 313-342.

VALPUESTA, V.; QUESADA, M.A.; SÂNCHEZROLDÁN; TIGER, H.A.; HEREDIA, A.; BUKOVAC, M.J. Changes in idole-3-acetic acid, idole3 -acetic acid oxidase, and peroxidase isoenzimes in the seeds of developing peach fruits. Journal Plant Growth Regulation, New York, v.8, p.255-261, 1989. 